01,13

\title{
Локализованные состояния вблизи тонкого слоя с нелинейными свойствами, разделяющего нелинейные фокусирующие и дефокусирующие среды
}

\section{(C) C.E. Савотченко}

Белгородский государственный технологический университет им. В.Г. Шухова, Белгород, Россия

E-mail: savotchenkose@mail.ru

(Поступила в Редакцию 28 мая 2018 г.

В окончательной редакции 20 ноября 2018 г.

Принята к публикации 14 ноября 2018 г.)

На основе нелинейного уравнения Шредингера описаны новые типы локализованных состояний вблизи контакта дефокусирующей и самофокусирующей сред с керровской нелинейностью, разделенных тонким дефектным слоем, характеризуемым нелинейностью такого же типа. Показано, что возникают два типа локализованных состояний. Получены дисперсионные соотношения, определяющие связь энергии локализации возбуждений с характеристиками сред и дефектного слоя. В явном аналитическом виде получены уровни энергии локализации в частных случаях.

DOI: $10.21883 /$ FTT.2019.04.47403.147

\section{1. Введение}

Локализованные состояния в нелинейных средах с дефектами различной физической природы изучаются теоретически и экспериментально достаточно давно и активно $[1,2]$. Наиболее разработанными с теоретической точки зрения в данном направлении являются вопросы существования локализованных состояний вблизи границ раздела сред, представляющих собой нелинейные поверхностные волны оптической [3], магнитной [4] и звуковой природы [5]. Локализация электрического и магнитного полей вдоль волноводов, роль которых играют плоские дефекты, границы раздела слоев, описывается нелинейными уравнениями как с керровской нелинейностью [6,7], так и с различными вариантами некерровской нелинейности [8,9].

Для теоретического описания различных нелинейных явлений и эффектов локализации возбуждений широко применяется нелинейное уравнение Шредингера (НУШ) [10,11], в частности, в случае локализации световых пучков перпендикулярно направлению их распространения в средах с керровской нелинейностью. Особое значение имеет изучение волноводных свойств границ раздела сред. Во многих случаях, особенно когда изучаются закономерности локализации оптических полей, в теоретических моделях используются граничные условия, соответствующие непрерывности поля и его тангенциальных компонент [6-11].

Если же влияние границ раздела сред, как плоских дефектов, имеет существенное значение, то возникает необходимость использования в модели характеристик границ, к примеру, интенсивности взаимодействия нелинейного возбуждения с плоскими дефектами, выступающими в роли волноводов [12-14]. Локализация нели- нейных возбуждений в двухуровневой системе, связанных на границе раздела линейной и нелинейной сред, рассматривалась в [15], а периодические состояния, возникающие в таких системах, проанализированы в [16]. Локализация нелинейных возбуждений в двухуровневой системе, которые линейным образом взаимодействуют с границей раздела нелинейных сред с противоположными знаками нелинейного отклика, была описана в [17].

Изучение особенностей локализации световых потоков вдоль волноводов часто требует введения дополнительных параметров, характеризующих взаимодействие электромагнитного поля с волноводом [18-23]. При слабой связи между волноводами амплитуда возбуждения в них может существенно превосходить среднюю амплитуду во всем кристалле, поэтому нелинейные слагаемые можно учитывать только внутри самих волноводов [18-20,23]. Важность изучения систем с такими свойствами обусловлена широкими возможностями использования плоскопараллельных волноводов с сильным взаимодействием с возбуждениями в нелинейной оптике. В качестве примера реалистичной физической системы, обладающей подобными оптическими свойствами, можно привести плоские волноводные структуры AlGaAs [24].

В настоящей работе использована модель нелинейного дефекта [18-22], представляющего собой плоский дефект, образованный при контакте дефокусирующей и самофокусирующей сред с керровской нелинейностью. В данной модели считается, что среды разделены тонким дефектным слоем, который внутри характеризуется также керровской нелинейностью.

Целью настоящей работы является определение новых типов локальных состояний и энергии локализации возбуждений вблизи нелинейного тонкого дефектного 
слоя с волноводными свойствами, возникающими за счет того, что среды по разные стороны от дефекта обладают противоположными знаками керровской нелинейности. Случай, когда нелинейный плоский дефект разделяет среды с одинаковыми знаками керровской нелинейности, был рассмотрен в [21], где показана возможность существования одного типа локальных состояний на границе раздела двух фокусирующих сред и трех типов локальных состояний на границе раздела двух дефокусирующих сред в различных энергетических диапазонах. В [22] показано, что в среде с самофокусировкой возникают новые типы периодических состояний, существование которых обусловлено нелинейностью дефекта и которые в случае линейного дефекта не возникают.

\section{2. Формулировка и уравнения модели}

Пусть граница раздела сред находится в плоскости $y z$ перпендикулярно оси $x$. Граница раздела как плоский дефект порождает возмущения характеристик среды. Такие возмущения сосредоточены на расстояниях существенно меньше ширины локализации распространяющихся в среде волн.

В $[25,26]$ было показано, что динамика светового поля в слоистой структуре описывается НУШ. Основываясь на данных результатах, рассмотрим систему плоскопараллельных чередующихся немагнитных узких и широких слоев, но теперь будем считать, что узкие слои характеризуются существенным нелинейным откликом.

Пусть ось $x$ направлена перпендикулярно плоскости слоев, а плоскость $y z$ параллельно им. В плоскополяризованной монохроматической волне, распространяющейся вдоль слоев, вектор напряженности электрического поля $\mathbf{E}$, направленного вдоль оси $y$, описывается уравнением Максвелла с показателем преломления, зависящим от координаты $x$ в поперечном слоям направлении: $n=n_{0}+n_{1}+n_{2}$, где $n_{0}$ и $n_{1}$ - линейные показатели преломления широких и узких слоев соответственно. Для сред с эффектом Керра нелинейный показатель преломления зависит от квадрата амплитуды поля: $n_{2}(x)=\{\alpha(x)+\beta(x)\}|\mathbf{E}|^{2}, \alpha(x)$ и $\beta(x)$ - коэффициенты керровской нелинейности сред в широких и узких слоях соответственно [23].

Монохроматическая волна с волновым вектором $\mathbf{k}=\mathbf{e}_{x} k_{z}\left(\mathbf{e}_{x}=\{1,0,0\}\right)$ и частотой $\omega_{0}=c k_{z} / n_{0}$ в адиабатическом приближении представима в виде:

$$
\mathbf{E}=\mathbf{e}_{y}\left\{E_{1}(x, t) \cos \left(k_{z} z-\omega_{0} t\right)+E_{2}(x, t) \sin \left(k_{z} z-\omega_{0} t\right)\right\},
$$

где $E_{2}$ и $E_{2}-$ медленно меняющиеся функции $x$ и $t$, $\mathbf{e}_{y}=\{0,1,0\}$. Для функции $\psi=E_{1}+i E_{2}$ при условиях $n_{1} \ll n_{0}, \alpha|\psi|^{2} \ll n_{0}$ и $\beta|\psi|^{2} \ll n_{0}$ в [25] было получено НУШ

$$
i \psi_{t}+A \psi_{x x}^{\prime \prime}+B \psi+\gamma|\psi|^{2} \psi=U \psi,
$$

где $A=\left(c / n_{0}\right)^{2} / 2 \omega_{0}, B=n_{1} \omega_{0} / n_{0}, \quad \gamma=\alpha \omega_{0} / n_{0}, U-$ потенциал, учитывающий различия показателей прелом- ления в узких и широких слоях. В пределе ультратонких слоев $(h \ll a, h-$ ширина узких слоев, $2 a-$ расстояние между ними), разделяющих широкие слои, в [25] этот потенциал аппроксимировался выражением с дельта-функцией Дирака.

Ограничимся случаем, когда один ультратонкий слой разделяет два оптических полупространства, которые занимают керровские среды с противоположными знаками нелинейного отклика. Граница раздела сред считается плоской и много меньше характерного масштаба локализации возмущений параметров среды, ей создаваемой. Теперь предлагается учесть нелинейность и внутри узких слоев. Тогда потенциал $U$ будет определяться коэффициентом керровской нелинейности внутри ультратонкого слоя $\beta$.

Учитывая приведенное НУШ, будем рассматривать взаимодействие нелинейных возбуждений, локализованных вблизи плоского дефекта, с нелинейными свойствами на основе одномерного НУШ традиционного вида

$$
i \psi_{t}^{\prime}=-\frac{1}{2 m} \psi_{x x}^{\prime \prime}+\Omega(x) \psi-\gamma(x)|\psi|^{2}+U(x) \psi,
$$

где $m=-1 / 2 A-$ „эффективная масса“ возбуждения, $\Omega=-B$. Будем считать, что среды по разные стороны от дефекта могут иметь различные линейные показатели преломления. Тогда можно положить

$$
\Omega(x)= \begin{cases}\Omega_{1}, & x<0 \\ \Omega_{2}, & x>0\end{cases}
$$

где величины $\Omega_{1,2}$ считаются всюду постоянными.

В настоящей работе рассматривается случай, когда среды по разные стороны от плоскости дефекта характеризуются ангармонизмом взаимодействия элементарных возбуждений с противоположными знаками: $\gamma(x)=-\gamma_{1}$, $x<0$ для дефокусирующей среды и $\gamma(x)=\gamma_{2}, x>0$ для самофокусирующей среды, где константы $\gamma_{1,2}>0$.

Следуя $[19,20]$, для описания плоского дефекта с нелинейными свойствами будем использовать потенциал в виде

$$
U(x)=U_{0} \delta(x)|\psi|^{2},
$$

где $\delta(x)$ - дельта-функция Дирака, $U_{0} \propto \beta / n_{0}-$ интенсивность нелинейного отклика дефекта такая, что при $U_{0}>0$ возбуждение отталкивается от дефекта, а при $U_{0}<0-$ притягивается.

Задача нахождения стационарных состояния с энергией $E$ сводится подстановкой в (1) волновой функции в виде $\psi(x, t)=\psi(x) \exp (-i E t)$, в результате чего получается стационарное НУШ

$$
E \psi=-\frac{1}{2 m} \psi_{x x}^{\prime \prime}+\Omega(x) \psi-\gamma(x)|\psi|^{2} \psi+U(x) \psi .
$$

В рассматриваемой физической интерпретации величина $E$ имеет смысл частоты нелинейной поверхностной волны, локализованной в поперечном границе разделу сред направлении. 
Нелинейное уравнение со слагаемым вида (2) использовалось при формулировке модели оптической системы, в которой периодическая модуляция линейного показателя преломления сочетается с одиночным нелинейным дефектом [23,27].

Решение НУШ (3) с потенциалом (2) сводится к решению НУШ без потенциала

$$
\psi_{x x}^{\prime \prime}+2 m\left(E-\Omega(x)+\gamma(x) \psi^{2}\right) \psi=0,
$$

с двумя граничными условиями сопряжения в плоскости дефекта при $x=0$ :

$$
\begin{gathered}
\psi(-0)=\psi(+0)=\psi(0), \\
\psi^{\prime}(+0)-\psi^{\prime}(-0)=2 m U_{0}|\psi(0)|^{2} .
\end{gathered}
$$

Если ввести амплитуду поля на границе раздела сред $\psi_{0}=\psi(-0)=\psi(+0)$, то граничное условие (6) примет вид:

$$
\psi^{\prime}(+0)-\psi^{\prime}(-0)=2 m U_{0}\left|\psi_{0}\right|^{2} .
$$

Следует отметить, что амплитуда нелинейных волн является важной характеристикой, поскольку от нее зависит энергия (частота) нелинейных стационарных состояний [1].

Как известно, типы решений НУШ определяются знаком нелинейности. Связанные на границе раздела их комбинации, соответствующие различным знакам нелинейности сред по разные от нее стороны, определяют типы нелинейных локализованных состояний.

\section{3. Нелинейные локализованные состояния}

В диапазоне $\Omega_{1}<E<\Omega_{2}$, что возможно при условии $\Omega_{1}<\Omega_{2}$, уравнение (4) имеет решение, удовлетворяющее граничным условиям (5) и (6), представимое в виде

$$
\psi(x)=\left\{\begin{array}{ll}
A_{t} \text { th } a_{t}\left(x-x_{1}\right), & x<0 \\
A_{2} / \operatorname{ch} q_{2}\left(x-x_{2}\right), & x>0
\end{array} .\right.
$$

Параметры $x_{1,2}$ характеризуют положения „центров“ связанных кинка и солитона по разные стороны от плоскости дефекта.

Подстановка (8) в (4) позволяет получить амплитуды

$$
\begin{gathered}
A_{t}^{2}=q_{t}^{2} /\left(m \gamma_{1}\right), \\
A_{2}^{2}=q_{2}^{2} /\left(m \gamma_{2}\right)
\end{gathered}
$$

и волновые числа

$$
\begin{gathered}
q_{t}^{2}=m\left(E-\Omega_{1}\right), \\
q_{2}^{2}=2 m\left(\Omega_{2}-E\right) .
\end{gathered}
$$

Из (11) и (12) следует связь между величинами $q_{t}$ и $q_{2}$

$$
q_{t}^{2}=m\left(\Omega_{2}-\Omega_{1}\right)-q_{2}^{2} / 2 .
$$

Значения энергии локализованного состояния определяются из соотношения, полученного после подстановки решения (8) в граничные условия (5) и (6)

$$
q_{2}=-\eta q_{t} \text { th } q_{t} x_{1} \operatorname{ch} q_{2} x_{2}
$$

$$
q_{2} \text { th } q_{2} x_{2}+2 q_{t} / \operatorname{sh} 2 q_{t} x_{t}=2 U_{0} q_{2}^{2} / \gamma_{2} \operatorname{ch}^{2} q_{2} x_{2},
$$

где $\eta=\left(\gamma_{2} / \gamma_{1}\right)^{1 / 2}$.

Таким образом, из соотношения (15), с учетом (13), получается волновое число, которое позволяет найти энергию как функцию параметров $E=E\left(m, U_{0}, \gamma_{1,2}, x_{2}\right)$. Из соотношения (14) затем находится $x_{1}$. Поэтому волновая функция (8) является однопараметрическим решением НУШ, характеризуемым одним свободным параметром, в качестве которого выбран $x_{2}$.

Если энергия возбуждения находится в диапазоне $E<\min \left\{\Omega_{1}, \Omega_{2}\right\}$, то НУШ (4) имеет решение вида

$$
\psi(x)=\left\{\begin{array}{ll}
A_{1} / \operatorname{sh} q_{1}\left(x-x_{1}\right), & x<0 \\
A_{2} / \operatorname{ch} q_{2}\left(x-x_{2}\right), & x>0
\end{array} .\right.
$$

Для ограниченности решения (16) должно выполняться требование: $x_{1}<0$.

Подстановка (16) в НУШ (4) позволяет получить амплитуду

$$
A_{1}^{2}=q_{1}^{2} /\left(m \gamma_{1}\right)
$$

и волновое число:

$$
q_{1}^{2}=2 m\left(\Omega_{1}-E\right) .
$$

Амплитуда $A_{2}$ определяется выражением (10), а волновое число $q_{2}$ определяется выражением (12).

Подстановка (16) в граничные условия (5) и (6) приводит к дисперсионным соотношениям

$$
\begin{gathered}
\eta q_{1} \operatorname{ch} q_{2} x_{2}=-q_{2} \operatorname{sh} q_{1} x_{1}, \\
q_{2} \text { th } q_{2} x_{2}-q_{1} \operatorname{cth} q_{1} x_{1}=2 U_{0} q_{2}^{2} / \gamma_{2} \operatorname{ch}^{2} q_{2} x_{2} .
\end{gathered}
$$

Соотношение (18) служит для нахождения волнового числа, позволяющего получить энергию как функцию параметров системы $E=E\left(m, U_{0}, \gamma_{1,2}, x_{2}\right)$. Затем из соотношения (17) можно определить параметр $x_{1}$. В качестве свободного параметра выбран $x_{2}$.

В некоторых частных случаях имеется возможность получить выражение для энергии локализованного состояния типа (16) в явном аналитическом виде.

\section{4. Условия локализации}

Сначала рассмотрим случай, когда $\Omega_{1}=\Omega_{2}=\Omega$, откуда следует, что $q_{1}=q_{2}=q$. Если рассмотреть решение специального вида при $x_{2}=0$, то из (18) получается волновое число

$$
q=-\frac{\gamma_{2} \sqrt{\eta^{2}+1}}{2 U_{0} \eta}
$$


которое позволяет определить энергию такого локализованного состояния в явном виде

$$
E=\Omega-\gamma_{2}\left(\gamma_{1}+\gamma_{2}\right) / 8 m U_{0}^{2} .
$$

Из (17) тогда с учетом (19) получается выражение для положения „центра““ солитона

$$
x_{1}=\frac{2 U_{0} \eta}{\gamma_{2} \sqrt{\eta^{2}+1}} \operatorname{Arsh} \eta .
$$

Для существования такого вида локализованного состояния с энергией (20) и „центром“ солитона (21) в силу требования ограниченности решения (16) должно выполняться условие $U_{0}<0$. Другими словами, рассматриваемое состояние может локализоваться только вблизи притягивающего дефекта.

Если рассмотреть теперь решение (16) с $\Omega_{1}=\Omega_{2}=\Omega$ и симметрично расположенными „центрами“ солитонов, когда $x_{1}=-x_{2}=-x_{0}$, из (18) получаем волновое число

$$
q=\frac{\gamma_{2}}{2 U_{0} \eta} \frac{1+\eta^{2}}{1-\eta^{2}}
$$

которое позволяет определить энергию такого локализованного состояния в явном виде

$$
E=\Omega-\frac{\gamma_{2}^{2}}{8 m U_{0}^{2} \eta^{2}}\left(\frac{1+\eta^{2}}{1-\eta^{2}}\right)^{2} .
$$

Из (17) тогда с учетом (22) получается выражение для положения „центра“ солитона

$$
x_{0}=\frac{2 U_{0} \eta}{\gamma_{2}} \frac{1-\eta^{2}}{1+\eta^{2}} \operatorname{Arth} \eta \text {. }
$$

Для ограниченности решения (16) $x_{0}$ должно быть положительным, поэтому такое состояние локализуется вблизи отталкивающего дефекта (то есть когда $U_{0}>0$ ).

Рассмотрим теперь случай, когда $\Omega_{1} \neq \Omega_{2}$ и $x_{2}=0$. Тогда из (17) и (18) получается энергия такого локализованного состояния в явном виде

$$
E=\Omega_{2}-\Omega_{a}\left\{1 \pm\left(1-\Omega_{b} / \Omega_{a}\right)^{1.2}\right\}
$$

где

$$
\begin{gathered}
\Omega_{a}=\gamma_{2}\left(\gamma_{1}+\gamma_{2}\right) / 16 m U_{0}^{2}, \\
\Omega_{b}=2 \gamma_{2}\left(\Omega_{2}-\Omega_{1}\right) /\left(\gamma_{1}+\gamma_{2}\right) .
\end{gathered}
$$

Локализованное состояние рассматриваемого вида с энергией (25) существует при выполнении условия: $\Omega_{2}-\left(\gamma_{1}+\gamma_{2}\right)^{2} / 32 m U_{0}^{2}<\Omega_{1}<\Omega_{2}$.

Наконец, рассмотрим случай, когда $\Omega_{1} \neq \Omega_{2}$, $x_{1} \neq x_{2} \neq 0$, и выполняется требование $q_{j} x_{j} \ll 1$. Такое условие можно назвать длинноволновым приближением. Оно описывает состояния, энергия которых близко расположена к краю спектра: $\left|\Omega_{j}-E\right| \ll 1 / 2 m x_{j}^{2}$. $\mathrm{B}$ рассматриваемом приближении характеристики локализованного состояния выражаются через свободный параметр $x_{2}$. Тогда из (17) получается положение $x_{1}=-\eta x_{2}$, а из (18) энергия такого локализованного состояния описывается выражением

$$
E=\Omega_{2}-\frac{\sqrt{\gamma_{1} \gamma_{2}}}{2 m x_{2}\left(2 U_{0}-\gamma_{2} x_{2}\right)}
$$

Такое локализованное состояние с энергией (26) существует, если выполняются требования: 1) $x_{2}>0$ и $U_{0}>\gamma_{2} x_{2} / 2$ (отталкивающий нелинейный дефект) или 2) $x_{2}<0$ и $U_{0}<\gamma_{2} x_{2} / 2$ (притягивающий нелинейный дефект).

\section{5. Заключение}

Следует отметить, что при $U_{0}=0$, то есть вблизи дефекта без нелинейного отклика, локальные состояния рассмотренных видов не реализуются. Это означает, что их существование обусловлено исключительно нелинейными свойствами дефекта.

Таким образом, в данной работе аналитически описаны особенности локализации возбуждений вблизи тонкого дефектного слоя с нелинейными свойствами, разделяющего среды с различными свойствами, в частности с различными характеристиками ангармонизма кристаллов противоположных знаков. Учет противоположных знаков нелинейности, характеризующих фокусирующие и дефокусирующие среды, позволил описать два новых типа локализованных состояний, дополняющих исследования [19-21].

Полученные результаты изучения нелинейных поверхностных волн, распространяющихся вдоль границ раздела сред с различными физическими характеристиками, имеют существенное значение в связи с их широким применением в оптических системах хранения данных $[28,29]$. Кроме того, особенности несимметричного распределения поля вдоль границ раздела сред следует учитывать при определении управляющих свойств таких интерфейсов, для которых возможны переключения режимов пропускания и запирания потоков поля [30,31].

\section{Список литературы}

[1] А.М. Косевич, А.С. Ковалев. Введение в нелинейную физическую механику. Наук. думка, Киев (1989). 304 с.

[2] Д. Михалаке, Р.Г. Назмитдинов, В.К. Федянин. Физика элементарных частиц и атомного ядра (ЭЧАЯ) 20, 198 (1989).

[3] Y.S. Kivshar, G.P. Agrawal. Optical Solitons: From Fibers to Photonic Crystals. Academic Press, San Diego (2003). 540 p.

[4] А.Б. Борисов, В.В. Киселев. Нелинейные волны, солитоны и локализованные структуры в магнетиках. Квазиодномерные магнитные солитоны. УрО РАН, Екатеринбург (2009). T. $1.512 \mathrm{c}$.

[5] В.И. Горенцвейг, Ю.С. Кившарь, А.М. Косевич, Е.С. Сыркин. ФНТ 16, 1472 (1990).

[6] Y.V. Bludov, D.A. Smirnova, Yu.S. Kivshar, N.M.R. Peres, M.I. Vasilevsky. Phys. Rev. B 89, 035406 (2014). 
[7] I.V. Shadrivov, A.A. Sukhorukov, Yu.S. Kivshar, A.A. Zharov, A.D. Boardman, P. Egan. Phys. Rev. E 69, 016617 (2004)

[8] Л.Ф. Федоров, К.Д. Ляхомская. Письма в ЖТФ 23, 36 (1997).

[9] О.В. Коровай, П.И. Хаджи. ФТТ 45, 364 (2003).

[10] F.Kh. Abdullaev, B.B. Baizakov, B.A. Umarov. Opt. Commun. 156, 341. (1998).

[11] Н.Н. Ахмедиев, В.И. Корнеев, Ю.В. Кузьменко. ЖЭТФ 88, 107 (1985).

[12] С.Е. Савотченко. Вестн. Воронежского гос. ун-та. Сер. Физика. Математика 4, 51 (2016).

[13] С.Е. Савотченко. Конденсированные среды и межфазные границы 19, 4, 567 (2017).

[14] С.Е. Савотченко. Вестн. Воронежского гос. ун-та. Сер. Физика. Математика 1, 44 (2018).

[15] С.Е. Савотченко. Конденсированные среды и межфазные границы 19, 2, 291 (2017).

[16] С.Е. Савотченко. ЖТФ 62, 1776 (2017).

[17] S.E. Savotchenko. Commun. Nonlinear Sci. Numer. Simul. 63, 171 (2018).

[18] I.V. Gerasimchuk. J. Nano-Electron. Phys. 4, 04024-1 (2012).

[19] I.V. Gerasimchuk, P.K. Gorbach, P.P. Dovhopolyi. Ukr. J. Phys. 57, 678 (2012).

[20] И.В. Герасимчук. ЖЭТФ 121, 596 (2015).

[21] S.E. Savotchenko. Mod. Phys. Lett. B 32, 1850120 (2018).

[22] С.Е. Савотченко. Письма в ЖЭТФ 107, 481 (2018).

[23] A.A. Sukhorukov, Y.S. Kivshar. Phys. Rev. Lett. 87, 083901 (2001).

[24] B. Luther-Davies, G.I. Stegeman. Spatial Optical Solitons. Springer-Verlag, N.Y. (2001).

[25] И.В. Герасимчук, А.С. Ковалев. ФНТ 26, 799 (2000).

[26] И.В. Герасимчук, А.С. Ковалев. ФТТ 45, 1088 (2003).

[27] Y.V. Kartashov, B.A. Malomed, L. Torner. Rev. Mod. Phys. 83, 247 (2011).

[28] I.S. Panyaev, N.N. Dadoenkova, Yu.S. Dadoenkova, I.A. Rozhleys, M. Krawczyk, I.L. Lyubchanckii, D.G. Sannikov. J. Phys. D 9, 435103 (2016).

[29] И.С. Паняев, Д.Г. Санников. Компьютерная оптика 41, 183 (2017).

[30] M.D. Tocci, M.J. Bloemer, M. Scalora, J.P. Dowling, C.M. Bowden. Appl. Phys. Lett. 66, 2324 (1995).

[31] S. Lan, H. Ishikawa. J. Appl. Phys. 91, 2573 (2002).

Редактор Ю.Э. Китаев 